\title{
Evolution of MLO-like proteins in flowering plants
}

\author{
Pozharskiy A.S. ${ }^{1,2 *}$, Gritsenko D.A. ${ }^{1,2}$ \\ ${ }^{1}$ Institute of Plant Biology and Biotechnology, Almaty, Kazakhstan \\ ${ }^{2}$ Al-Farabi Kazakh National University, Almaty, Kazakhstan \\ *email: aspozharsky@gmail.com
}

MLO-like proteins (MLP) are the family of transmembrane proteins in plants. The first MLP was discovered in barley as a factor of susceptibility to mildew, and its numerous homologs were found in various groups of plants, from algae to angiosperms. Studies have shown contribution of MLP to susceptibility to fungal and bacterial diseases in other species as well, including Arabidopsis thaliana, Solanum lycopersicum, etc. MLP are assumed to be targets for specific plant-pathogen interactions, and loss-of-function mutations have been shown to increase resistance to corre. The scope of the present work is to investigate phylogenetic relations across wide range of MLP sequences known up to date.

MLP sequences belonging to green plants were retrieved from UniProt database and preliminary clustered using CD-HIT with similarity threshold $50 \%$. In order to exclude individual highly variable proteins, only proteins included into clusters with 10 or more sequences were selected for further analysis (2749 in total). The final set of sequences included MLP from flowering plants and 5 accessions from gymnosperms as an outgroup. Analysis included multiple sequence alignment (MAFFT), identification and selection of conserved regions (UGENE), building Maximum Likelihood (MEGA-X) and Neighbor-Joining trees (R/phangorn), motif search (MEME).

Tree structures revealed by ML and NJ methods were consistent between each other. Groups of homologs were observed at the different taxonomic levels. Three principal clusters and five sub-clusters were observed at the level of angiosperms in general. Inside sub-clusters a clear distinction between super-order clades and to some extent between orders was observed. Positions of the most represented orders were in accordance to their known phylogeny (APG IV). Characteristic amino-acid motifs were found to describe distinct clusters and sub-cluster groups.

Revealed phylogenetic structure can be useful for classification of MLP family across wide range of plant species. Distinction of parallel orthologous lines will help to put existing studies of MLP and their impact on plants into wider systematic context. 